\title{
Infrared Scattering-Type Scanning Near-Field Optical Microscopy of Biomembranes in Water
}

Emanuel Pfitzner, Joachim Heberle*

Department of Physics, Experimental Molecular Biophysics, Freie Universität Berlin, 14195 Berlin, Germany

*corresponding author: joachim.heberle@fu-berlin.de

Supporting text contains details about experimental methods and the phase correction procedure applied in Figure $2 \mathrm{C}, \mathrm{D}, \mathrm{G}, \& \mathrm{H}$ of the main text. Supporting Figure 1 and 2 contain near-field maps for demodulation orders $n=1,2, \& 3$ for tip or sample scanning. Supporting Figure 3 shows lines extracted from the phase maps shown in Figure 2 in the main text to derive the signal-to-noise ratio. Supporting Figure 4 shows line profiles extracted from the near-field maps of purple membrane patches to derive the spatial resolution. Supporting Figure 5 shows the raw data for Figure 3 in the main text and necessary phase correction. Supporting Figure 6 shows the extent of interference fringes in the very central field of view.

\section{Supporting Text}

Bottom-illumination scheme. The IR SSNOM setup has been described elsewhere. ${ }^{1}$ The presented bottom-illumination scheme SSNOM is based on a NanoWizard II (JPK Instruments AG, Germany) equipped with a $x-y$ sample scanner stage (TAO). A silicon hemisphere ( $\varnothing=10 \mathrm{~mm}$, AMS Technologies AG, Germany) is fit centrally into a home-built 
aluminum plate and fixed by a PEEK gasket. One part of the collimated light from an attenuated external cavity quantum cascade laser (QCL, Daylight Solutions, USA) is guided through a beamsplitter (1" ZnSe window, Eksma Optics UAB, Lithuania) into the entrance pupil of a 40x Schwarzschild objective (LMM-40x-P01, 40x reflective objective, $0.5 \mathrm{NA}$, infinity corrected back focal plane, Thorlabs $\mathrm{GmbH}$, Germany) and focused through the Si hemisphere onto the Pt-coated AFM tip (Arrow NCPt, NanoAndMore GmbH, Germany) oscillating at $\Omega \sim 280 \mathrm{kHz}$ in air or $\Omega \sim 130 \mathrm{kHz}$ in $\mathrm{H}_{2} \mathrm{O}$. The scattered light is guided back to the ZnSe beamsplitter and is directed onto a mercury-cadmium-telluride photodetector (KLD0.1-J1/DC/11, Kolmar Technologies Inc., USA) where the light is interferometrically detected and fed into a lock-in amplifier (HF2LI, Zurich Instruments, Switzerland). The signal is then analyzed using a pseudo-heterodyne detection scheme as previously described. ${ }^{2}$ All nearfield maps are postprocessed to retrieve the full $-\pi$ to $\pi$ phase information. ${ }^{3}$ Phase maps were then corrected by subtracting the median of the phase line-by-line. Phase maps in Figure $2 \mathrm{D}, \mathrm{E}, \mathrm{G}, \& \mathrm{H}$ are subjected to a more elaborate line-by-line phase correction algorithm (see below).

Laser-scanning assisted sSNOM. A pair of $5 \mathrm{~mm}$ galvanometric scan mirrors (GVS102, 2D Galvo System, gold coated, Thorlabs GmbH, Germany) was installed in between the ZnSe beamsplitter and the objective to track the scanning tip with the IR focus. The scanned beam was relayed by a pair of telecentric lenses (LA5040, 1/2" uncoated $\mathrm{CaF}_{2}$ lens, $f=40 \mathrm{~mm}$, Thorlabs $\mathrm{GmbH}$, Germany) and imaged into the entrance pupil of the objective. The auxiliary outputs of the $x-y$ scanner are scaled and offset by a homebuilt circuit and fed into the controller of the 2D galvanometric scan mirror to accurately match the beam-scanning with the tip/sample scanning.

Sample preparation. Purple membrane was kindly provided by the group of Dr. Ramona Schlesinger (Genetic Biophysics, Freie Universität Berlin, Germany). Before each experiment the Si hemisphere was cleansed by stepwise sonication in acetone, isopropanol and pure water for at least $15 \mathrm{~min}$. Purple membrane patches were diluted to $10 \mu \mathrm{g} / \mathrm{ml}$ in a buffered solution (300 mM KCl, $10 \mathrm{mM}$ Tris, $\mathrm{pH} 7.8$, filtered through $0.2 \mu \mathrm{m}$ filter) according to ref. ${ }^{4}$. 
$50 \mu \mathrm{l}$ of this solution was incubated for $30 \mathrm{~min}$ on the flat face of the hemisphere to allow the patches to adsorb. The hemisphere was then gently rinsed with pure water subsequently and dried. For experiments in an aqueous environment, $200-500 \mu \mathrm{l}$ of a solution of $150 \mathrm{mM} \mathrm{KCl}$, $10 \mathrm{mM}$ Tris, $\mathrm{pH} 7.8$ (filtered through $0.2 \mu \mathrm{m}$ filter) were added to the surface.

Line-by-line Phase Correction. All near-field phase maps recorded with this technique can, in principle, suffer from offsets in the phase by drift of the optical path lengths of both the tip arm and the reference arm. To compensate that, all maps, unless noted, are corrected by subtracting the median of the phase of each line. In Figure $2 \mathrm{D}, \mathrm{E}, \mathrm{G}, \& \mathrm{H}$ the phase has been corrected line-by-line by the following protocol: for each line $i$ the pixels $j$ with a near-field amplitude $s_{i}(j)$ larger than a threshold $s_{i}(j)>\max _{j}\left\{s_{i}(j)\right\}$ are selected. The phase $\varphi_{i}(j)$ of the corresponding pixels $j$ is then fitted either by a constant or linear function and subtracted from all phase values in the line $i$. If necessary, a plane was fitted to the phase data and subtracted. 


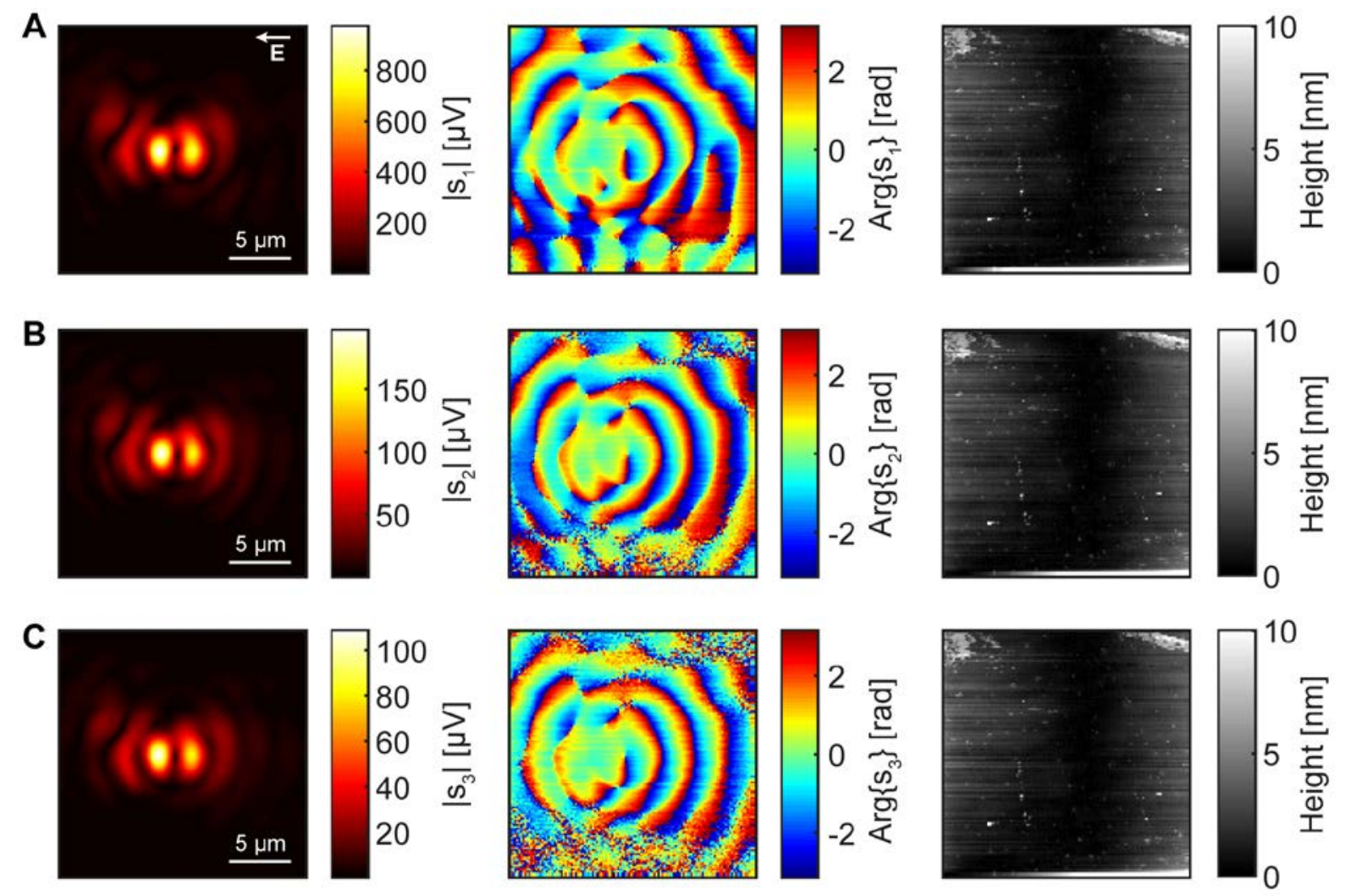

Supporting Figure 1 Near-field distributions for all harmonics (tip scanning). Near-field amplitude, phase and topography (left to right) for harmonics $n=1(\mathbf{A}), n=2(B)$, and $n=3(\mathbf{C})$. The white arrow indicates the incident polarization. 


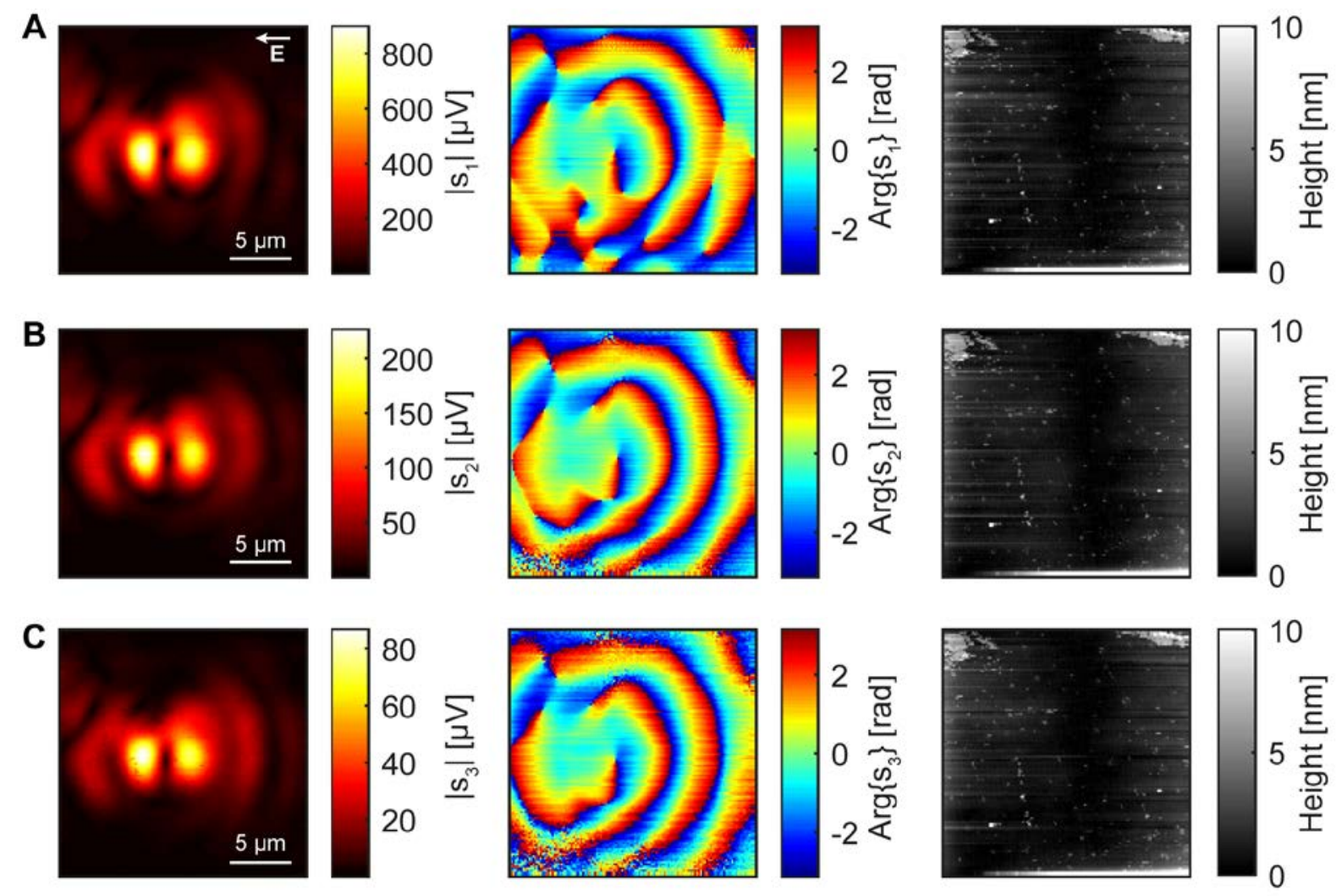

Supporting Figure 2 Near-field distributions for all harmonics (sample scanning). Near-field amplitude, phase and topography (left to right) for harmonics $n=1(\mathbf{A}), n=2(B)$, and $n=3(\mathbf{C})$. The white arrow in the top left panel indicates the incident polarization. Please note, that the scale bar is the same as in supporting figure 1. 


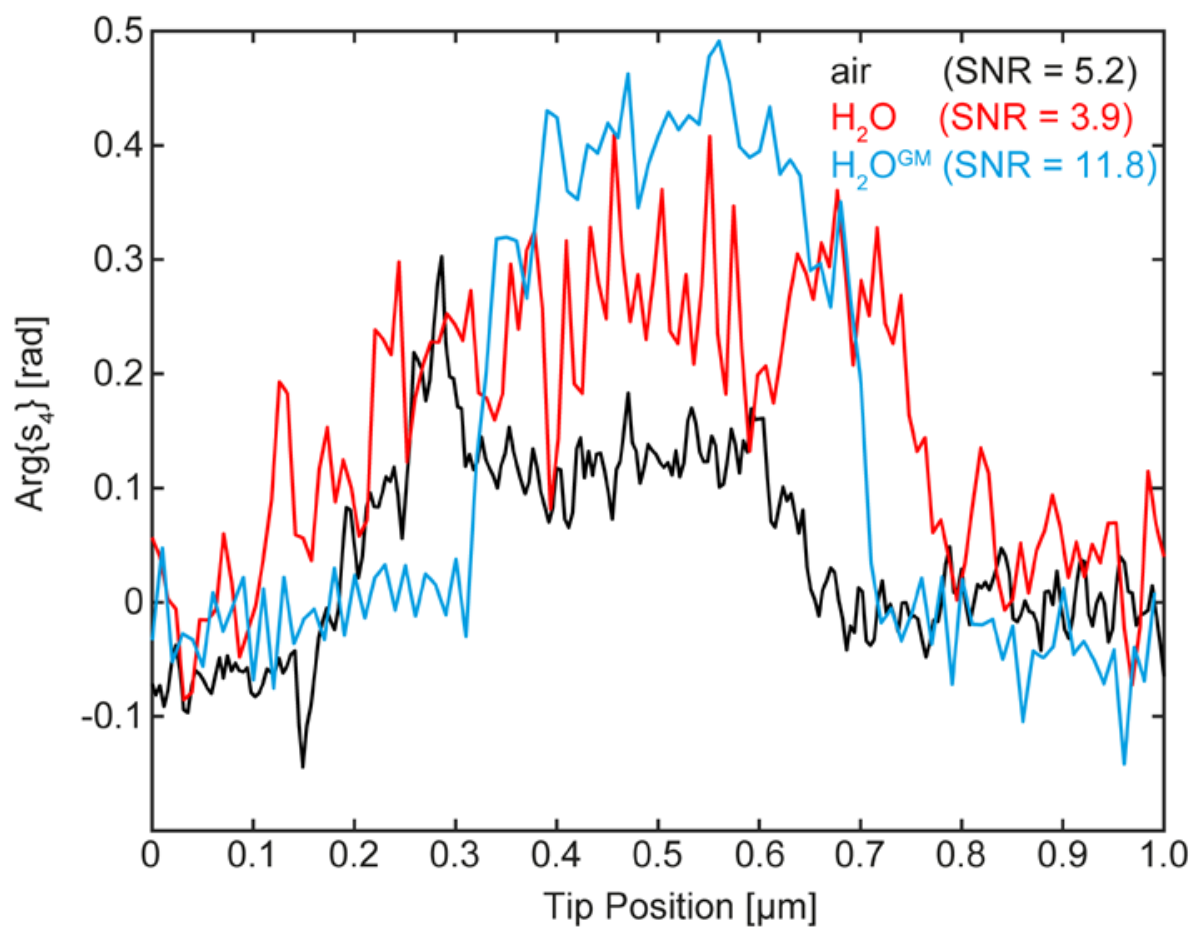

Supporting Figure 3 Signal-to-noise determination. Traces show the fluctuations during the line scans which have been extracted from the phase maps shown in Figure 2D\&G and Figure 4B in the main text. The black and red traces correspond to recordings in air and in $\mathrm{H}_{2} \mathrm{O}$, respectively, the blue trace to recordings in $\mathrm{H} 2 \mathrm{O}$ with the galvanometric scan mirror modification. 

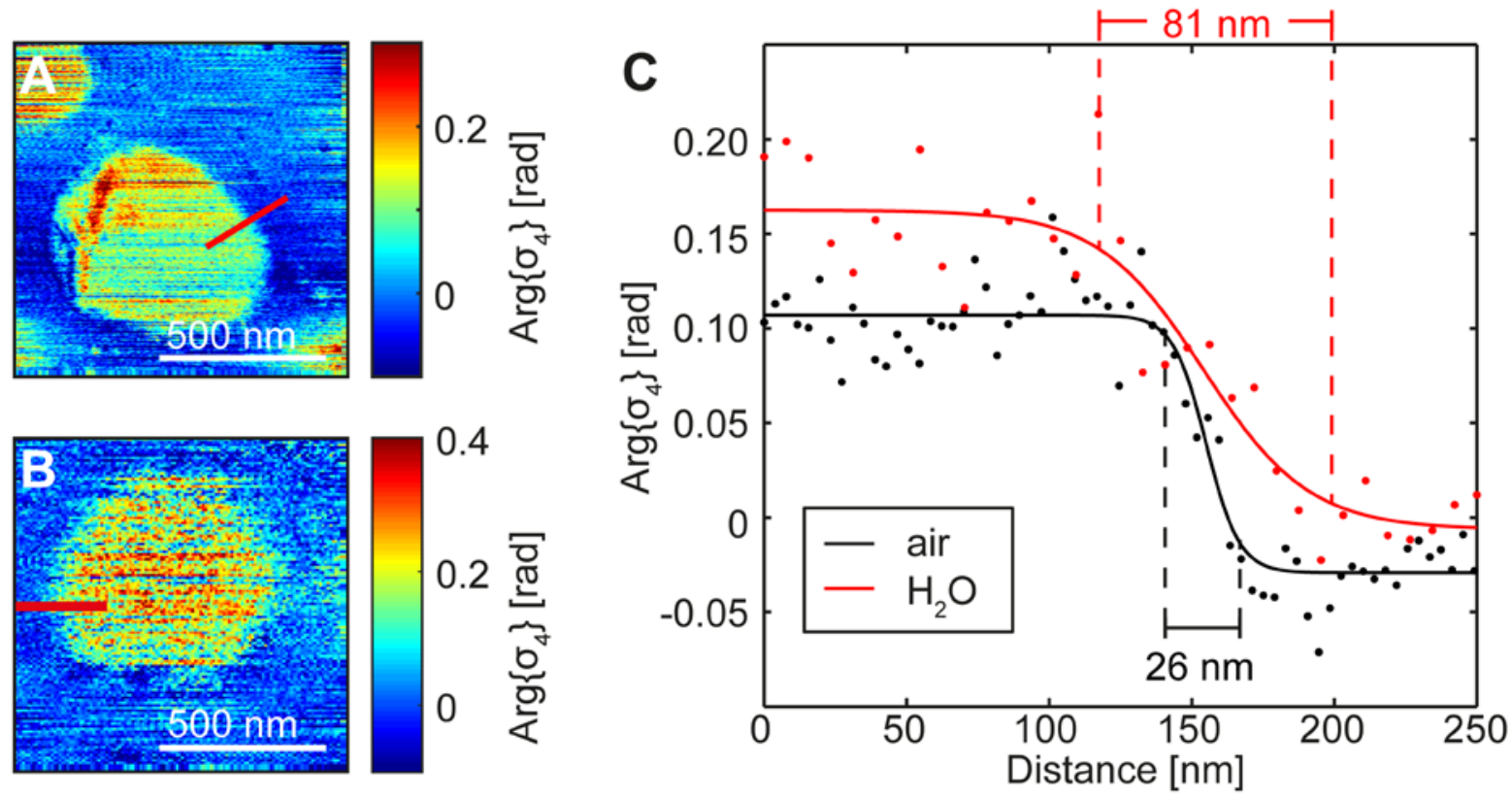

Supporting Figure 4 Line profiles extracted from near-field phase maps. A-B Near-field phase maps of a PM patch (identical to Figure 2 in the main text) in air (A) and in $\mathrm{H}_{2} \mathrm{O}(\mathbf{B})$ Maps have been recorded with the QCL emission tuned to $1659 \mathrm{~cm}^{-1}$. The red line indicates the line along which the line profile was extracted. C Extracted line profiles (average of three adjacent lines) as indicated in $\mathbf{A}$ and $\mathbf{B}$ for maps recorded in air (black trace) and $\mathrm{H}_{2} \mathrm{O}$ (red trace). The solid line indicates a sigmoidal fit to the extracted traces. The distance for the phase to drop from $90 \%$ to $10 \%$ of the sigmoidal fit is $\Delta \mathrm{X}_{\text {air }}=26( \pm 12) \mathrm{nm}$ and $\Delta \mathrm{X}_{\mathrm{H} 2 \mathrm{O}}=81( \pm$ 46) $\mathrm{nm}$, respectively. 

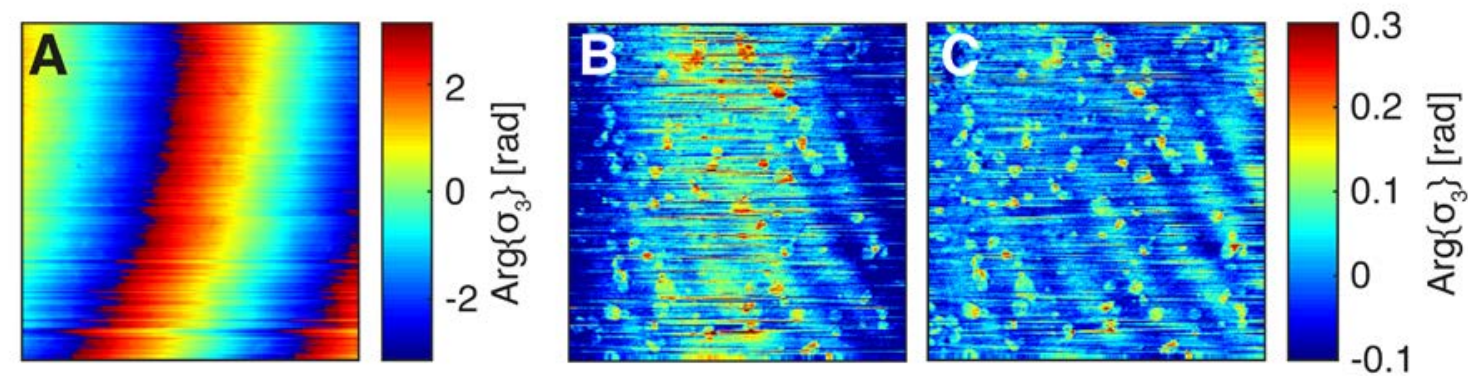

Supporting Figure 5 Phase correction of wide field data. A-C Near-field phase of a 20x20 $\mu \mathrm{m}^{2}$ scan while tracing the tip with the galvano mirrors. A No phase correction applied, B line-by-line linear phase subtracted \& $\mathbf{C}$ line-by-line quadratic phase subtracted. 


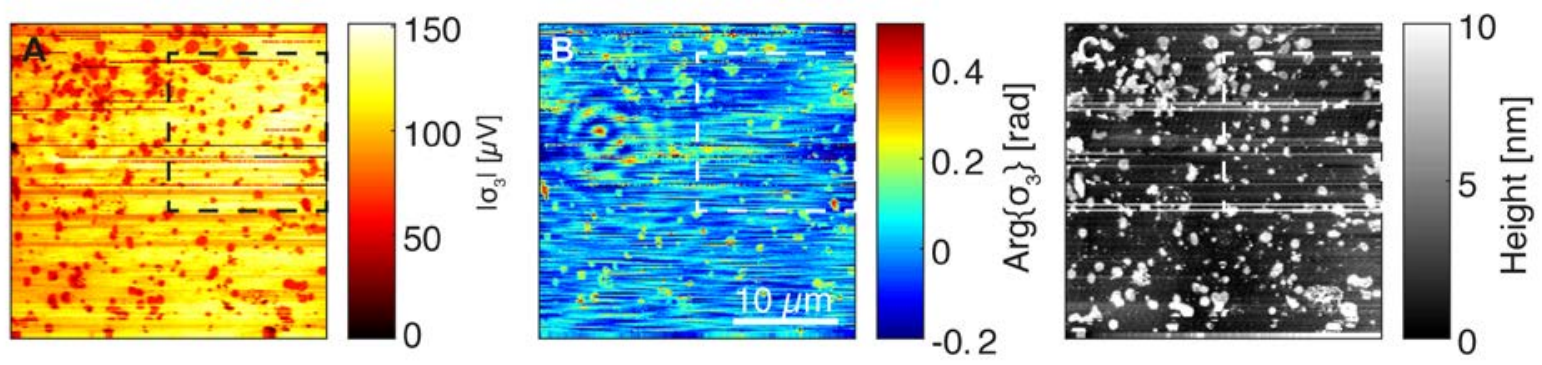

Supporting Figure 6 Central Fringes in $30 \times 30 \mu m^{2}$ field of view. A-C Near-field amplitude (A), phase (B) and topography (C) of a $30 \times 30 \mu \mathrm{m}^{2}$ scan while tracing the tip with the galvanometric scan mirrors. The dashed box indicates the field of view shown in Figure 3 in the main text. Interference fringes are visible in the upper right area due to light reflected by the spherical face of the hemisphere. The stripe-like artifacts are due to particles temporarily sticking to the probing tip and decreasing the optical signal. 


\section{Supplementary References}

1. Mészáros, L. S.; Ceccaldi, P.; Lorenzi, M.; Redman, H. J.; Pfitzner, E.; Heberle, J.; Senger, M.; Stripp, S. T.; Berggren, G. Spectroscopic Investigations under Whole-Cell Conditions Provide New Insight into the Metal Hydride Chemistry of [FeFe]-Hydrogenase. Chem. Sci. 2020, 11, 4608-4617.

2. Ocelic, N.; Huber, A.; Hillenbrand, R. Pseudoheterodyne Detection for BackgroundFree Near-Field Spectroscopy. Appl. Phys. Lett. 2006, 89, 101124.

3. Moreno, C.; Alda, J.; Kinzel, E.; Boreman, G. Phase Imaging and Detection in Pseudo-Heterodyne Scattering Scanning Near-Field Optical Microscopy Measurements. Appl. Opt. 2017, 56, 1037-1045.

4. Müller, D. J.; Engel, A. Atomic Force Microscopy and Spectroscopy of Native Membrane Proteins. Nat. Protoc. 2007, 2, 2191-2197. 